\title{
Electric Dipole Moments of Acetone and of Acetic Acid Measured in Supersonic Expansion
}

\author{
O. Dorosh AND Z. KISIEL*
}

Institute of Physics, Polish Academy of Sciences

al. Lotników 32/46, 02-668 Warszawa, Poland

Dedicated to the memory of Professor Jerzy Prochorow

\begin{abstract}
Precise values for electric dipole moments of isolated acetone and acetic acid molecules were determined from the Stark effect measurements made on lowest $J$ rotational transitions at conditions of supersonic expansion. The new results are $\mu_{\text {tot }}=\mu_{b}=2.9345(22) \mathrm{D}$ for acetone from measurements on the $1_{11} \leftarrow 1_{01}$ transition, and $\mu_{a}=0.8631(8), \mu_{b}=1.4345(11), \mu_{\text {tot }}=$ 1.6741(10) $\mathrm{D}$ for acetic acid, from measurements on $1_{11} \leftarrow 0_{00}$ and $1_{01} \leftarrow 0_{00}$ transitions. A comparison with previous determinations and with results of quantum chemical calculations is presented.
\end{abstract}

PACS numbers: 31.15.Ar, 32.10.Dk, 32.30.Bv, 32.60.+i, 33.15.Kr, 33.1.Mt, 33.20.Bx, 33.20.Sn

\section{Introduction}

Electric dipole moment is an important molecular property that gives insight into reactivity of molecules, in particular into the relative molecular orientations in early stages of chemical reactions. In addition, since intensity of rotational spectra is directly dependent on the magnitude of molecular dipole moment, precise knowledge of this quantity is of direct importance in quantitative determination of molecular abundances under atmospheric and astrophysical conditions probed by means of rotational spectroscopy. The principal technique for determination of electric dipole moments of isolated molecules has been the investigation of the Stark effect exhibited by rotational transitions of molecules subjected to an external electric field [1]. For some molecules there may be complications arising from the necessity to include in the Hamiltonian a description of additional effects such as nuclear hyperfine coupling or internal rotation. As an example, rotational spectra of two well known molecules, acetone $\left(\mathrm{CH}_{3}\right)_{2} \mathrm{CO}$, with two methyl internal rotors [2], and acetic acid $\mathrm{CH}_{3} \mathrm{COOH}$, with one methyl internal rotor [3], have

*corresponding author; e-mail: kisiel@ifpan.edu.pl 


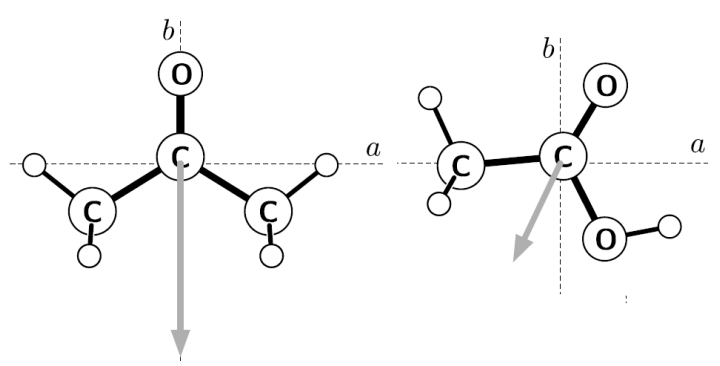

Fig. 1. The orientation of the molecule and of its electric dipole moment in the inertial frame for acetone (left) and acetic acid (right). The dipole moment vectors are directed from the notional negative to the notional positive charge, and are drawn to the same scale for the two molecules.

been thoroughly analysed only recently. Both molecules, see Fig. 1, are currently of astrophysical interest, with lines of both acetone [4] and acetic acid [5] detected in the interstellar medium. Although results of several Stark effect investigations have been reported for acetone [6,7] and acetic acid [8], those are now fairly dated and in need of revisiting with contemporary methods. We presently take advantage of the new knowledge of the rotational spectra of these two molecules and of improved experimental techniques of the Stark measurements in order to determine precise values for the dipole moments of acetone and of acetic acid.

\section{Experimental}

All measurements were made at conditions of supersonic expansion with the cavity Fourier transform microwave (FTMW) spectrometer in Warsaw [9], which is a coaxial microwave component version of the original Balle-Flygare design [10]. Measurements were characterised by rotational temperatures of less than $1 \mathrm{~K}$ and by transition frequency accuracy of better than $2 \mathrm{kHz}$. The expansion mixture was prepared by using vapour from above liquid samples of the studied substances over which Ar carrier gas was passed at a backing pressure of $1.1 \mathrm{~atm}$. A T-type sample inlet arrangement containing two sample tubes was positioned just upstream of the expansion nozzle. Control of expansion gas composition was possible by regulating the gas flow over the two sample tubes, one of which was in this case empty and acted as a bypass. The sample was pulsed through a $0.35 \mathrm{~mm}$ diameter orifice in a modified General Valve Series 9 valve, at a rate of $2-5 \mathrm{~Hz}$, and in a direction at right angle to the longitudinal axis of the microwave cavity.

The Stark measurements were carried out with the electrode arrangement shown in Fig. 2, which was designed in our laboratory to provide improved electric field uniformity under the specific requirements of the cavity FTMW experiment $[11,12]$. In order to avoid perturbing the microwave mode in the Fabry-Perot resonator the electrodes have to be relatively far apart, in our case by $27 \mathrm{~cm}$. The dimensions of the high-vacuum chamber require that electrode size cannot 
be much greater than their separation. With standard parallel plate electrodes such conditions lead to considerable spillage of the electric field outside the interelectrode region, and bending of field lines in the interelectrode region [11]. The resulting loss in electric field uniformity is such that the expanding molecules are subjected to an electric field that is on average only $95 \%$ of that given by the standard $\Delta V / d$ relation for infinite parallel plate electrodes, separated by distance $d$ and charged to potential difference $\Delta V$. This was realised relatively early on $[13,14]$, and resulted in varying instrumental solutions, including the use of guard wires and resistors for the perpendicular FTMW configuration [15], and guard rings [16] for the parallel configuration. Our solution [11] uses small guard electrodes attached to the sides of the parallel plates, that focus the electric field to close to the $\Delta V / d$ condition and lead to considerable improvement in field uniformity. The dimensions of the electrodes were established by three-dimensional electric field calculations [11], and their operation confirmed by actual spectroscopic calibration [12]. The final solution is robust in that the electrodes can be easily and reproducibly assembled within the spectrometer chamber, and there are no separators between electrodes, which could collect electric charge and distort the electric field.

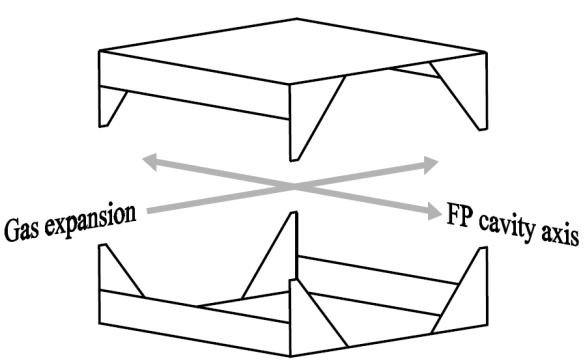

Fig. 2. The Stark electrode geometry developed at IFPAN [11] in order to ensure a uniform electric field at the large electrode separation in relation to their size that is enforced by the experimental constraints of the supersonic expansion FTMW experiments. The main plates of the electrodes are $28 \times 28 \mathrm{~cm}$ in size and are separated by $27 \mathrm{~cm}$.

The large electrode separation enforces Stark measurements at relatively low values of the electric field. For safety reasons we usually limit the potential difference between the electrodes to $10000 \mathrm{~V}$ (applied as $\pm 5000 \mathrm{~V}$ relative to ground), which corresponds to field strength of only $370 \mathrm{~V} / \mathrm{cm}$. For the $J=1 \leftarrow 0$ transition of the standard calibrant molecule, OCS, this corresponds to the Stark shift of only $0.8 \mathrm{MHz}$ for the $\Delta M=0$ component. For this reason we adopted a calibration procedure based on the $J=1 \leftarrow 0$ transitions of two different symmetric top molecules, $\mathrm{CH}_{3} \mathrm{I}$ and $\mathrm{CH}_{3} \mathrm{CN}[11,12]$. Both transitions exhibit Stark shifts of at least $5 \mathrm{MHz}$ at the field strength at our disposal. 
The procedure also has the advantage of providing a number of cross-checks since Stark measurements are made on all three hyperfine components of each transition: $F=5 / 2 \leftarrow 5 / 2,7 / 2 \leftarrow 5 / 2,3 / 2 \leftarrow 5 / 2$ at $14694.931,15100.749$, $15275.894 \mathrm{MHz}$, respectively, for $\mathrm{CH}_{3} \mathrm{I}$, and $F=1 \leftarrow 1,2 \leftarrow 1,0 \leftarrow 1$ at 18396.725, 18397.996, 18399.892 $\mathrm{MHz}$, respectively, for $\mathrm{CH}_{3} \mathrm{CN}$. Although any given Stark measurement is nominally rather precise it is rarely appreciated that there are many experimental factors that may differ between different measurements. One such factor are the expansion conditions, which depend on backing pressure, sample concentration, volatility, and expansion thermodynamics. These will certainly differ between the calibrant and the molecule under study leading to some differences in the dimensions of the supersonic expansion plume and its interaction with the microwave cavity mode. The dimensions of the Fabry-Perot mode also depend on the microwave frequency and on the mirror separation, both of which will unavoidably vary between different measurements. In the present case we determined effective electrode separation of 26.956(3) $\mathrm{cm}$ with $\mathrm{CH}_{3} \mathrm{I}$, and 26.946(2) $\mathrm{cm}$ with $\mathrm{CH}_{3} \mathrm{CN}$. These two values differ by more than their nominal statistical error, which is evidence for the small but measurable role of the various effects discussed above. In further work we have, therefore, used the average of these values, $26.95(2) \mathrm{cm}$, in which the uncertainty has been increased to a level that is expected to realistically account for the various experimental uncertainties. Calibrations and dipole moment determinations were carried out with program QSTARK $[11,17]$, which dispenses with the various approximations used in such treatments by setting up and diagonalising the complete Hamiltonian matrix for each value of the applied electric field and of the $M$ quantum number.

\section{Results and analysis}

The analysis of results of Stark measurements normally draws on the complete knowledge of the rotational spectrum of the molecule. The pertinent energy levels are calculated from assumed values of spectroscopic constants in the rotational Hamiltonian and the value of the dipole moment is the only parameter of fit. In the two title molecules the ground torsional state is split into several substates, $A$ and $E$ for acetic acid, and $A A, E E, E A$, and $A E$ for acetone. The analysis in $[2,3]$ was aimed at a global fit of all relevant substates by means of specialised internal rotation Hamiltonians. The resulting constants of the pure rotational part of each Hamiltonian are listed in Table I, in the columns marked "global" constants. It should be realised that these constants constitute only a small subset of around 40 adjustable parameters used in the internal rotation fits for acetone and acetic acid $[2,3]$. It is known, however, that the actual ground state, namely substate $A$ in acetic acid, and substate $A A$ in acetone, can be satisfactorily treated with the standard asymmetric rotor Hamiltonian [18]. In such case the contributions from internal rotation are folded into "effective" values of constants in the rotational Hamiltonian, also listed in Table I. In the latter fits some sextic and 
higher centrifugal distortion constants were required in order to obtain a fit down to experimental accuracy, but those higher order constants have no effect on low$J$ transitions used in the dipole moment determination. The differences between the global and effective constants are in some cases quite appreciable, and arise from sizable torsional contributions due to low internal rotation barriers in the two molecules, 251(3) $\mathrm{cm}^{-1}$ [19] in acetone and $170.2 \mathrm{~cm}^{-1}$ [3] in acetic acid.

TABLE I

Spectroscopic constants in Watson's $A$-reduced Hamiltonian in representation $I^{\mathrm{r}}$ [18] for acetone and acetic acid.

\begin{tabular}{l|c|c|c|c}
\hline \hline \multirow{2}{*}{} & \multicolumn{2}{|c|}{$\left(\mathrm{CH}_{3}\right)_{2} \mathrm{CO}$} & \multicolumn{2}{c}{$\mathrm{CH}_{3} \mathrm{COOH}$} \\
\cline { 2 - 5 } & global $^{a}$ & effective $^{b}$ & global $^{a}$ & $\begin{array}{c}\text { effective }^{b} \\
\text { Ref. [3] }\end{array}$ \\
& Ref. [2] & $A A$-state & $11324.034(21)$ & $11415.32024(19)$ \\
\hline$A[\mathrm{MHz}]$ & $10165.21654(80)$ & $10186.2451(11)$ & $9494.190(6)$ & $9477.68426(10)$ \\
$B[\mathrm{MHz}]$ & $8515.16477(65)$ & $8519.94925(99)$ & $5326.1497(21)$ & $5326.335647(92)$ \\
$C[\mathrm{MHz}]$ & $4910.19903(44)$ & $4910.12529(61)$ & $4.2924(12)$ & $4.38976(13)$ \\
$\Delta_{J}[\mathrm{kHz}]$ & $4.9055(25)$ & $5.4253(37)$ & $10.466(6)$ & $9.9109(14)$ \\
$\Delta_{J K}[\mathrm{kHz}]$ & $-3.620(17)$ & $10.518(18)$ & $-0.177(18)$ & $138.9979(47)$ \\
$\Delta_{K}[\mathrm{kHz}]$ & $10.245(17)$ & $20.337(19)$ & $1.7199(6)$ & $1.778993(55)$ \\
$\delta_{J}[\mathrm{kHz}]$ & $2.0645(12)$ & $2.3254(18)$ & $10.536(24)$ & $4.25977(78)$ \\
$\delta_{K}[\mathrm{kHz}]$ & $-0.7393(56)$ & $7.8887(80)$ & & 0.0445 \\
$\sigma_{\text {fit }}[\mathrm{MHz}]$ & & 0.203 & & 0.810 \\
$\sigma_{\mathrm{w}}^{c}$ & & 1.279 & 2518 & 1109 \\
$N_{\text {lines }}$ & 1002 & 267 & & \\
\hline
\end{tabular}

${ }^{a}$ From a full internal rotation Hamiltonian fitted to all four substates of the ground torsional state in acetone, and the two pairs of substates for the ground and first excited torsional states in acetic acid.

${ }^{b}$ Resulting from a refit made in this work of only the $A A$ substate transitions in acetone from Ref. [2], and the $A$ substate transitions in acetic acid from Ref. [3].

${ }^{c}$ Weighted deviation of fit, $\sigma_{\mathrm{w}}=\left[N_{\text {degf }}^{-1} \sum_{i}\left(\left(\nu_{\text {obs }}^{i}-\nu_{\text {calc }}^{i}\right) / \delta \nu_{i}\right)^{2}\right]^{1 / 2}$.

Rotational constants of both molecules are sufficiently large that the strongest rotational transitions in the frequency range of the spectrometer are the $J=1 \leftarrow 0$ transitions, which all fall in the $14.8-16.8 \mathrm{GHz}$ region, and are very suitable for the Stark measurements because of simple Stark behaviour. In addition, for acetic acid both ${ }^{a} R$ - and ${ }^{b} R$-type transitions are intense facilitating determination of both $\mu_{a}$ and $\mu_{b}$ dipole moment components. These features have already been taken advantage of previously $[6,8]$. The torsional energy differences in acetone, $E_{E E-A A}=2.3 \mathrm{GHz}, E_{E A-A A}=E_{A E-A A}=4.6 \mathrm{GHz}$ [19], and in acetic acid, $E_{E-A}=11.0 \mathrm{GHz}^{\dagger}$, are also such that there will be no torsionally

${ }^{\dagger}$ Estimated from the data in [3] using the procedures and tabulations from Ref. [20]. 
excited rotational levels that could be sufficiently close in energy to the studied energy levels to perturb the single state Stark behaviour. The effective constants from Table I are therefore expected to provide an accurate basis for describing the rotational contribution to the energies of the Stark components.

TABLE II

The results of the Stark measurements for acetone and acetic acid.

\begin{tabular}{|c|c|c|c|c|c|}
\hline \multicolumn{3}{|c|}{$\left(\mathrm{CH}_{3}\right)_{2} \mathrm{CO}$} & \multicolumn{3}{|c|}{$\mathrm{CH}_{3} \mathrm{COOH}$} \\
\hline$V^{a}[\mathrm{~V}]$ & obs. $[\mathrm{MHz}]$ & $\mathrm{o}-\mathrm{c}[\mathrm{kHz}]$ & $V^{a}[\mathrm{~V}]$ & obs. [MHz] & $\mathrm{o}-\mathrm{c}[\mathrm{kHz}]$ \\
\hline \multicolumn{3}{|c|}{$1_{11} \leftarrow 0_{00}, M=0 \leftarrow 0$} & \multicolumn{3}{|c|}{$1_{01} \leftarrow 0_{00}, M=0 \leftarrow 0$} \\
\hline 0.0 & 15096.3314 & -1.0 & 0.0 & 14804.0020 & -0.4 \\
\hline 2601.1 & 15097.0355 & 0.8 & 3750.9 & 14804.2634 & 3.3 \\
\hline 3548.0 & 15097.6413 & 2.2 & 5174.6 & 14804.4932 & 0.2 \\
\hline 4628.5 & 15098.5594 & 3.3 & 6664.3 & 14804.8172 & 1.2 \\
\hline 5263.3 & 15099.2091 & 1.3 & 7587.8 & 14805.0578 & 0.7 \\
\hline 6570.2 & 15100.8153 & 2.6 & 8476.7 & 14805.3170 & -1.7 \\
\hline 7631.4 & 15102.3756 & -0.9 & 9272.7 & 14805.5770 & -0.4 \\
\hline 8483.3 & 15103.7976 & -3.2 & 9728.2 & 14805.7361 & 0.2 \\
\hline \multicolumn{3}{|c|}{$1_{11} \leftarrow 0_{00}, M=1 \leftarrow 0$} & \multicolumn{3}{|c|}{$1_{11} \leftarrow 0_{00}, M=0 \leftarrow 0$} \\
\hline 2601.1 & 15096.6366 & 1.9 & 0.0 & 16741.4952 & 0.1 \\
\hline 3548.0 & 15096.8967 & 1.8 & 3750.9 & 16741.8640 & 1.2 \\
\hline \multirow[t]{6}{*}{4628.5} & 15097.2910 & 1.4 & 5174.6 & 16742.1946 & -0.3 \\
\hline & & & 6664.3 & 16742.6564 & 0.6 \\
\hline & & & 7587.8 & 16742.9998 & 0.0 \\
\hline & & & 8476.7 & 16743.3723 & -0.7 \\
\hline & & & 9272.7 & 16743.7426 & 0.4 \\
\hline & & & 9728.2 & 16743.9681 & -0.3 \\
\hline \multicolumn{3}{|c|}{$\begin{array}{c}\mu_{b}=2.93450(28) \mathrm{D} \\
\sigma_{\text {fit }}=2.1 \mathrm{kHz}\end{array}$} & \multicolumn{3}{|c|}{$\begin{array}{c}\mu_{a}=0.86307(41) \mathrm{D}^{b} \\
\mu_{b}=1.43445(28) \mathrm{D}^{b} \\
\sigma_{\text {fit }}=1.1 \mathrm{kHz}\end{array}$} \\
\hline
\end{tabular}

${ }^{a}$ The voltage difference between the Stark electrodes, which was converted to electric field by using the experimentally determined effective electrode separation of $26.95 \mathrm{~cm}$.

${ }^{b}$ The cited uncertainty is the standard error from the fit, which does not yet account for the uncertainty in field calibration, see text.

The results of the Stark measurements made for both molecules are summarised in Table II, and in the form of the Stark shift plots in Fig. 3 and Fig. 4. The Stark behaviour appears in all cases to be close to the second order Stark-effect limit. In our experiment the external electric field was applied in direc- 


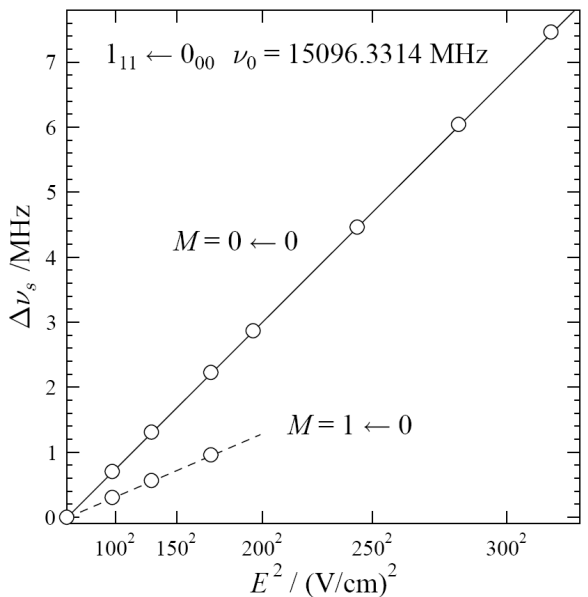

Fig. 3. Graphical summary of Stark shift measurements for acetone, made on the ${ }^{b} R$ type $1_{11} \leftarrow 0_{00}$ rotational transition. Circles denote experimental points and the lines are drawn on the basis of the fitted dipole moment.

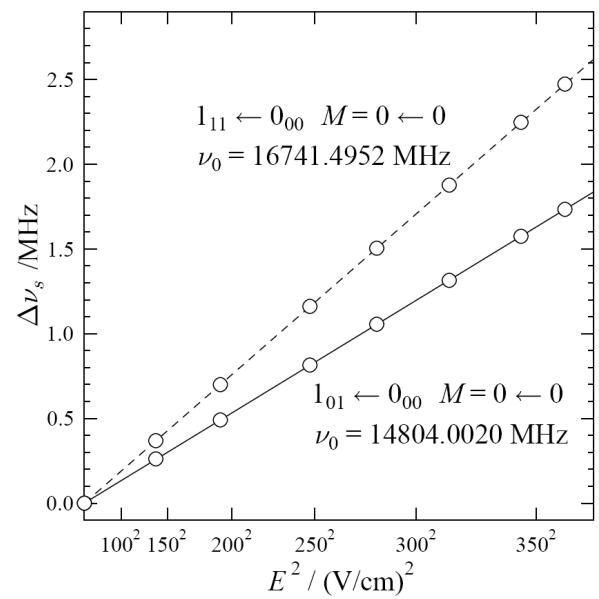

Fig. 4. Graphical summary of Stark shift measurements for acetic acid, made on the ${ }^{b} R$-type $1_{11} \leftarrow 0_{00}$ rotational transition, and the ${ }^{a} R$-type $1_{01} \leftarrow 0_{00}$ transition. Circles denote experimental points and the lines are drawn on the basis of the fitted dipole moment components.

tion parallel to the vector of the microwave radiation, so that only the $\Delta M=0$ Stark components would be expected to be observed. Nevertheless, small perturbations in the cylindrical symmetry of the Fabry-Perot resonator sometimes allow $\Delta M= \pm 1$ components to be measured at the same time [12], and an example of such measurement is also reported for acetone. The results of the Stark measurements were fitted to close to the experimental accuracy of the spectrometer, 
TABLE III

Comparison between experimental and calculated electric dipole moments (Debye) for acetone and acetic acid.

\begin{tabular}{|c|c|c|c|c|}
\hline & \multirow{2}{*}{$\begin{array}{l}\left(\mathrm{CH}_{3}\right)_{2} \mathrm{CO} \\
\mu_{\mathrm{tot}}=\mu_{b}\end{array}$} & \multicolumn{3}{|c|}{$\mathrm{CH}_{3} \mathrm{COOH}$} \\
\hline & & $\mu_{a}$ & $\mu_{b}$ & $\mu_{\text {tot }}$ \\
\hline this work & $2.9345(22)$ & $0.8631(8)$ & $1.4345(11)$ & $1.6741(10)$ \\
\hline literature & $2.93(3)^{a}$ & $0.86(1)^{b}$ & $1.47(2)^{b}$ & $1.70(2)^{b}$ \\
\hline B3LYP $/ 6-31 G(d, p)^{c}$ & 2.829 & 0.692 & 1.448 & 1.605 \\
\hline $\mathrm{MP} 2 / 6-31 \mathrm{G}(\mathrm{d}, \mathrm{p})$ & 2.720 & 0.654 & 1.293 & 1.449 \\
\hline B3LYP/6-311G $++(d, p)$ & 3.139 & 0.861 & 1.502 & 1.732 \\
\hline $\mathrm{MP} 2 / 6-311 \mathrm{G}++(\mathrm{d}, \mathrm{p})$ & 2.937 & 0.795 & 1.304 & 1.527 \\
\hline B3LYP/aug-cc-pVDZ ${ }^{d}$ & 3.095 & 0.923 & 1.544 & 1.799 \\
\hline MP2/aug-cc-pVDZ & 2.975 & 0.901 & 1.377 & 1.646 \\
\hline \multicolumn{5}{|l|}{${ }^{a}$ Ref. [7]. } \\
\hline \multicolumn{5}{|l|}{${ }^{b}$ Ref. [8]. } \\
\hline \multicolumn{5}{|c|}{$\begin{array}{l}{ }^{c} \text { All calculated values were obtained with the PC-GAMESS implemen- } \\
\text { tation [21] of the GAMESS package [22]. }\end{array}$} \\
\hline
\end{tabular}

with standard deviation of fit of $2.1 \mathrm{kHz}$ for acetone and $1.1 \mathrm{kHz}$ for acetic acid, by using only the dipole moment components as adjustable parameters. Table II reports the derived values for the dipole moment components together with the standard error resulting only from the statistics of the fit. The final values, accounting also for the uncertainty in field calibration, are reported in Table III. It is noted that the uncertainty in field calibration is the dominant source of experimental error in the final values, increasing their uncertainty by a factor ranging from five to an order of magnitude. This is an often neglected property of the Stark measurements at conditions of supersonic expansion, although it has been accounted for in all previous works of this type carried out in our laboratory, for example [24-26]. Finally a test was made also of the alternative way of dealing with the Stark analysis, by using the "global" constants from Table I. These are the "true" constants for the rotational part of the Hamiltonian, although they reproduce transition frequencies accurately only when used with constants that account for the torsional contributions. In this case we could, however, use the facility of fitting not the absolute frequencies but only the Stark shifts that is also built into the QSTARK program. The deviations of fit obtained in this way are slightly worse $(2.7 \mathrm{kHz}$ for acetone and $1.2 \mathrm{kHz}$ for acetic acid) and the derived values for the $\mu_{b}$ dipole moments are lower by $0.0045 \mathrm{D}$. Thus the sensitivity of the calculated Stark behaviour to differing assumptions is not large, at about the $2-3 \sigma$ level. Our preferred procedure of dipole moment evaluation is, nonetheless, expected to provide the cleanest reduction of the complex internal rotation be- 
haviour into a version compatible with the QSTARK program, although it might not necessarily be applicable for higher- $J$ transitions.

\section{Conclusions}

The present work reports hitherto the most precise values for ground state electric dipole moments of acetone and of acetic acid. The increase in precision of determination relative to the current literature values is more than an order of magnitude. The experimental values are also compared in Table III with values from typical levels of DFT and ab initio calculations, that would be used for molecules of this size. We note that for a given basis the listed DFT results are systematically greater than the MP2 values, and that the MP2/aug-cc-pVDZ level offers the closest reproduction of the experimental values, at better than $0.05 \mathrm{D}$ on average. It is noted that for any basis the DFT-MP2 difference of $0.1-0.15 \mathrm{D}$ is much greater than that closer to $0.01 \mathrm{D}$ found in calibration of computational results for the benchmark $\mathrm{HCl}$ molecule [27]. In addition, for $\mathrm{HCl}$ the switch to the aug-cc-pVTZ basis offered a factor of three reduction in the difference between calculated and experimental values but this was not found to be the case for the present molecules. However, since the number of basis functions already exceeded 300 , we only carried out an MP2/aug-cc-pVTZ calculation at the MP2/aug-ccpVDZ geometry, which resulted in practically the same dipole moment value as for the latter.

\section{Acknowledgments}

It has been a privilege for the corresponding author (Z.K.) to be able to interact with Professor Jerzy Prochorow on many different planes of science and administration of science over a period of more than 25 years. The enduring memory from all these encounters is one of the highest professionalism exhibited by Prof. Prochorow in dealing with the issues put before him, at a level that a typical scientist probably has little hope of reaching, but which he should always try to aspire to.

\section{References}

[1] W. Gordy, R.L. Cook, Microwave Molecular Spectra, Wiley, New York 1984.

[2] P. Groner, S. Albert, E. Herbst, F.C. De Lucia, F.J. Lovas, B.J. Drouin, J.C. Pearson, Astrophys. J. Suppl. Ser. 142, 145 (2002).

[3] V.V. Ilyushin, E.A. Alekseev, S.F. Dyubko, S.V. Podnos, I. Kleiner, L. Margules, G. Wlodarczak, J. Demaison, J. Cosleou, B. Mate, E.N. Karyakin, G.Yu. Golubiatnikov, G.T. Fraser, R.D. Suenram, J.T. Hougen, J. Mol. Spectrosc. 205, 286 (2001).

[4] F. Combes, M. Gerin, A.S. Wootten, G. Wlodarczak, F. Clausset, P.J. Encrenaz, Astron. Astrophys. 180, L13 (1987).

[5] D.M. Mehringer, L.E. Snyder, Y. Miao, F.J. Lovas, Astrophys. J. 480, L71 (1997). 
[6] J.D. Swalen, C.C. Costain, J. Chem. Phys. 31, 1562 (1959).

[7] R. Peter, H. Dreizler, Z. Naturforsch. 20a, 301 (1965).

[8] L.C. Krisher, E. Saegebarth, J. Chem. Phys. 54, 4553 (1971).

[9] Z. Kisiel, J. Kosarzewski, L. Pszczółkowski, Acta Phys. Pol. A 92, 507 (1997).

[10] T.J. Balle, W.H. Flygare, Rev. Sci. Instrum. 52, 33 (1981).

[11] Z. Kisiel, J. Kosarzewski, B.A. Pietrewicz, L. Pszczółkowski, Chem. Phys. Lett. 325, 523 (2000).

[12] Z. Kisiel, E. Białkowska-Jaworska, O. Desyatnyk, B.A. Pietrewicz, L. Pszczółkowski, J. Mol. Spectrosc. 208, 113 (2001).

[13] L.H. Coudert, F.J. Lovas, R.D. Suenram, J.T. Hougen, J. Chem. Phys. 87, 6290 (1987).

[14] M. Canagaratna, M.E. Ott, K.R. Leopold, Chem. Phys. Lett. 281, 63 (1997).

[15] T. Emilsson, H.S. Gutowsky, G. de Oliveira, C.E. Dykstra, J. Chem. Phys. 112, 1287 (2000).

[16] M. Schnell, D. Banser, J.-U. Grabow, Rev. Sci. Instrum. 75, 2111 (2004).

[17] Z. Kisiel, PROSPE - Programs for ROtational SPEctroscopy, available from http://info.ifpan.edu.pl/ kisiel/prospe.htm.

[18] J.K.G. Watson in: Vibrational Spectra and Structure, Ed. J.R. Durig, Vol. 6, Elsevier, New York 1977, p. 1.

[19] P. Groner, J. Mol. Struct. 550-551, 473 (2000).

[20] D.R. Herschbach, J. Chem. Phys. 31, 91 (1959).

[21] A.A. Granovsky, PC-GAMESS program, available from http://classic.chem.msu.su/gran/gamess/index.html.

[22] M.W. Schmidt, K.K. Baldridge, J.A. Boatz, S.T. Elbert, M.S. Gordon, J.H. Jensen, S. Koseki, N. Matsunaga, K.A. Nguyen, S.J. Su, T.L. Windus, M. Dupuis, J.A. Montgomery, J. Comput. Chem. 14, 1347 (1993).

[23] Extensible Computational Chemistry Environment Basis Set Database, developed and distributed by the Molecular Science Computing Facility, Environmental and Molecular Sciences Laboratory which is part of the Pacific Northwest Laboratory, P.O. Box 999, Richland, Washington 99352, USA, and funded by the U.S. Department of Energy, available from http://www.emsl.pnl.gov/forms/basisform.html.

[24] Z. Kisiel, O. Desyatnyk, L. Pszczółkowski, S.B. Charnley, P. Ehrenfreund, J. Mol. Spectrosc. 217, 115 (2003).

[25] Z. Kisiel, O. Desyatnyk, E. Białkowska-Jaworska, L. Pszczółkowski, Phys. Chem. Chem. Phys. 5, 820 (2003).

[26] O. Desyatnyk, L. Pszczółkowski, S. Thorwirth, T.M. Krygowski, Z. Kisiel, Phys. Chem. Chem. Phys. 7, 1708 (2005); 7, 2080 (2005).

[27] E. Białkowska-Jaworska, Z. Kisiel, L. Pszczółkowski, J. Mol. Spectrosc. 238, 72 (2006) 